\title{
Pharmacokinetics and Bioequivalence Evaluation of Two Voriconazole tablets: an Open-Label, Single-Dose, Randomized, Two-Way Crossover Study in Healthy Chinese Male Volunteers
}

\author{
Xuewen Qiu, Rongfeng Xiang, Qing Dai, Bo Yang, Lirong Xiong, Yangjing Ou, Min Tang, Yongchuan Chen*
}

Department of Pharmacy, Southwest hospital, Chongqing, China, 40003

\begin{abstract}
The aim of this study was to assess and compare the pharmacokinetic properties, bioavailability, and bioequivalence of a newly developed tablet of voriconazole with those of an established branded formulation in healthy Chinese adult male volunteers.

An open-label, single-dose, randomized, 2-way crossover study was conducted in fasted healthy Chinese male volunteers. Eligible participants were randomly assigned in a 1:1 ratio to receive one tablet $(200 \mathrm{mg})$ of the test or reference formulations, followed by a 1-week washout period and administration of the alternate formulations. Plasma samples were collected over 36 hours and analyzed by HPLC. The Voriconazole plasma concentration-time curves were used to obtain pharmacokinetic parameters including $A \cup C_{0-t}, A \cup C_{0-\infty}, C_{\max }$ and $T_{\max }$. The criteria for bioequivalence were $90 \% \mathrm{Cls}$ of $80 \%$ to $125 \%$ for AUC and $70 \%$ to $143 \%$ for $\mathrm{C}_{\text {max }}$, and no significant differences for $\mathrm{T}_{\text {max }}$ with a non-parameter test, according to guidelines of the SFDA of China. Tolerability was based on the recording of Adverse Events (AEs).

A total of 19 volunteers were included in the study. The mean (SD) $C_{\text {max }}, T_{m a x}, A U C_{0-t}$, and $A U C_{0-\infty}$ values after administration of the test and reference formulation, respectively, were as follows: $925.73(356.11)$ versus $1040.25(448.93) \mathrm{ng} / \mathrm{mL}, 1.57(0.98)$ vs 1.38(0.96) hours, 5304.97(3072.25) vs 5141.63(2976.92) ng/mL/h, and $5783.21(3266.86)$ vs $5520.69(3148.42) \mathrm{ng} / \mathrm{mL} / \mathrm{h}$. The relative bioavailability of the test formulation was $103.2 \%$

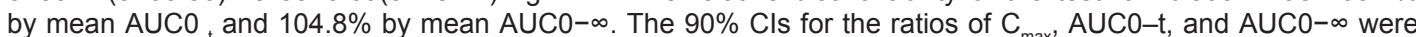
$77.3 \%$ to $122.7 \%, 85.7 \%$ to $114.3 \%$, and $83.6 \%$ to $116.4 \%$, respectively, meeting the predetermined criteria for bioequivalence. No drug-related AEs were observed.

In this study the test and reference formulations had similar PK parameters and similar plasma concentrationtime profiles and the test formulations met the regulatory criteria for bioequivalence to the established reference formulations based on the rate and extent of absorption. Both formulations were well tolerated.
\end{abstract}

Keywords: Bioequivalence; Pharmacokinetics; Voriconazole; HPLC

\section{Introduction}

Voriconazole is a broad-spectrum triazole antifungal agent with activity against a wide range of yeasts and filamentous fungi $[1,2]$ and is approved for treatment of adult and paediatric patients with invasive aspergillosis, those with fluconazole-resistant invasive Candida infections, non-neutropenic patients with candidaemia and those with emerging infections caused by Scedosporium species and Fusarium species.

Voriconazole exhibits nonlinear pharmacokinetics due to saturation of its metabolism. Although the pharmacokinetic (PK) characteristics and clinical pharmacology of voriconazole have been studied previously [3-5] insufficient data are available in a Chinese population. The present study was designed to assess and compare the PK parameters, bioavailability, and bioequivalence of the new voriconazole tablet versus the conventional tablet administered as single $200 \mathrm{mg}$ doses to healthy Chinese adult male volunteers.

\section{Subjects and Methods}

\section{Products and participants}

Voriconazole tablets (Vfend ${ }^{\mathrm{TM}} 200 \mathrm{mg}$; lot no. B10002040), purchased from Pfizer Pharmaceuticals Co. Ltd., and voriconazole tablets (200 mg; lot no. 100302), manufactured and provided by Chengdu Huashen Pharmaceuticals Co. Ltd. (Chengdu, People's
Republic of China), were used as the reference and test products, respectively, in assessing bioequivalence.

Healthy Chinese adult male volunteers participated in this study, which was conducted at Chongqing Southwest Hospital of the Third Military Medical University. Only male volunteers were enrolled because of the additional variables involved when studying women (eg, menstrual cycles and pregnancy), because guidelines of the State Food and Drug Administration (SFDA) of the People's Republic of China recommend using male volunteers [6].

Exclusion criteria included the administration of any medication (including over-the-counter remedies) 2 weeks before or during the study period. Physical examination ascertained that all volunteers were free of significant cardiac, hepatic, renal, pulmonary, neurologic,

*Corresponding author: Yongchuan Chen, Department of Pharmacy, Southwest hospital, 29TH, Gaotangyan Street, Shapingba District, Chongqing, China, 40003 Tel/Fax: 0086- 023-68754462; E-mail: zwmcyc@163.com

Received April 19, 2012; Accepted May 19, 2012; Published May 21, 2012

Citation: Qiu X, Xiang R, Dai Q, Yang B, Xiong L, et al. (2012) Pharmacokinetics and Bioequivalence Evaluation of Two Voriconazole tablets: an Open-Label, Single-Dose, Randomized, Two-Way Crossover Study in Healthy Chinese Male Volunteers. J Bioequiv Availab 4: 044-047. doi:10.4172/jbb.1000110

Copyright: (c) 2012 Qiu X, et al. This is an open-access article distributed under the terms of the Creative Commons Attribution License, which permits unrestricted use, distribution, and reproduction in any medium, provided the original author and source are credited. 
Citation: Qiu X, Xiang R, Dai Q, Yang B, Xiong L, et al. (2012) Pharmacokinetics and Bioequivalence Evaluation of Two Voriconazole tablets: an Open-Label, Single-Dose, Randomized, Two-Way Crossover Study in Healthy Chinese Male Volunteers. J Bioequiv Availab 4: $044-047$. doi:10.4172/jbb.1000110

gastrointestinal, and hematologic diseases. Volunteers were also examined by electrocardiography and laboratory tests such as hematology (complete blood count), urinalysis, biochemistry (total bilirubin, direct bilirubin, aspartate amino transferase, alanine amino transferase, alkaline phosphatase, plasma albumin, sodium, potassium, calcium, fasting blood glucose, blood uric acid, blood urea nitrogen plasma creatinine, triglycerides, and cholesterol), hepatitis B surface antigen, and HIV antibodies.

The present study was conducted in accordance with the ethical standards for studies in humans of the Declaration of Helsinki and its amendments [7] and the Guideline for Good Clinical Principles recommended by the SFDA [6]. The study protocol and informed consent form were approved by the ethics committee of Chongqing Southwest Hospital. All participants were informed by a clinical investigator of the study's aim and risks, and each submitted written informed consent before participating in the study.

\section{Study design}

This was an open-label, single-dose, randomized, 2-way crossover bioequivalence study with a 1-week washout period between each administration. Each volunteer received a single dose of either the test or reference product of $200 \mathrm{mg}$ voriconazole (one $200 \mathrm{mg}$ tablet), in random order based on computer-generated tables of random numbers. Each volunteer sample had a unique number, and personnel conducting the analyses were blinded to the group assignment.

After an overnight fast of 10 hours, the volunteers received 200 $\mathrm{mg}$ of either product of voriconazole (reference or test), taken with $200 \mathrm{~mL}$ of water. Additional water intake was permitted 2 hours after treatment, and food intake was allowed 4 hours after treatment. Alcoholic beverages, intense physical activity and smoking were not allowed during the study. Chinese regulatory authorities do not require testing of food effects in bioequivalence studies (as opposed to PK studies of Phase I clinical trials). In this study, food intake was strictly controlled and all volunteers received the same standardized food to minimize the effects of food on the study outcomes.

The volunteers were under continuous medical supervision at the controlled site throughout the study. Blood samples of $\sim 4 \mathrm{~mL}$ were drawn; the plasma was separated by centrifugation at $3000 \mathrm{rpm}$ for 5 minutes, followed by direct transfer into $2-\mathrm{mL}$ polypropylene tubes and storage at $-30^{\circ} \mathrm{C}$ until analysis. After a 1-week washout period, the study was repeated in the same manner to complete the crossover design.

\section{Tolerability}

Safety and tolerability were assessed frequently in each study by physical examinations, vital signs; laboratory measurements (including hematology, serum chemistry tests, routine urinalysis and scatoscopy) and electrocardiogram were also performed at baseline and completion of the study. Clinical adverse events, including systemic adverse events and those occurring locally at the SC injection site, were monitored throughout the study. Investigators evaluated all adverse events in terms of intensity (mild, moderate or severe), duration, outcome and relationship to study drug (none, remote, possible, probable or definitely related). The physician was not blinded to treatment.

\section{Measurement of plasma}

Plasma voriconazole concentrations were determined using validated high performance liquid chromatography (HPLC; Agilent 1100 Series). A Diamonsil C18 (2) $(250 \times 4.6$ mm, $5 \mu \mathrm{m})($ DikmaCo.,
Ltd., USA) was used for chromatographic separation. Voriconazole standard was purchased from the National Institute for the Control of Pharmaceutical and Biological Products. Carbamazepine was used as an internal standard for analytes, which was provided by the National Institute for the Control of Pharmaceutical and Biological Products. The mobile phase was acetonitrile: water: glacial acetic acid: triethylamine $=$ 42: 58: 0.1: 0.05. The flow rate of mobile phase was $1.4 \mathrm{~mL} / \mathrm{min}$ at room temperature, and the injection volume was $20 \mu \mathrm{L}$. Sample analysis involved calibration curves and QC samples at different concentrations to determine linearity, intra and inter day precision and accuracy, limit of quantification, selectivity, stability, and recovery.

\section{Pharmacokinetic and statistical analysis}

Pharmacokinetic parameters were analyzed by non-compartmental PK data analysis using Winnolin Version 5.2.1(Pharsight Corporation, mountainView, CA, USA). Bioequivalence of the two voriconazole formulations was assessed by calculating individual AUC0-t, $\mathrm{AUC}_{0-\infty}$, $\mathrm{C}_{\max }$ and $\mathrm{T}_{\max }$ values. The ratios (test $v s$ reference) of log-transformed data, together with their means and $90 \%$ CIs, were evaluated by analysis of variance (ANOVA) using Statistical Program for Social Sciences Version 16.0 (IBM, USA). If the $90 \%$ CI for AUC was located within $80 \%$ to $125 \%, \mathrm{C}_{\max }$ was within $70 \%$ to $143 \%$ of the statistical interval proposed by the SFDA, and no significant differences for $\mathrm{T}_{\text {max }}$ with a non-parameter test, the two formulations would be considered bioequivalent.

\section{Results}

\section{Subjects}

20 healthy Chinese male volunteers were enrolled in the study. One subject voluntarily withdrew during the second period. 19 participants (mean [SD] age, 22.6 [2.3] years [range, 20-27 years]; weight, 58.1 [4.6] $\mathrm{kg}$ [range, 53.0-78.0 kg] and height, 170.8 [4.7] $\mathrm{cm}$ [range, 161.0-181.0 $\mathrm{cm}$ ) completed the study and were included in the PK analysis at the last. No clinically significant AEs were observed during or after the study.

\section{Methodology result}

No significant interference at the retention times of voriconazole and IS was observed on chromatography of blank plasma. The observed recovery of voriconazole was within $87.22 \%$ to $105.87 \%$.The calibration curve, established by plotting the peak area ratio (y) versus concentration (x), was linear over the range of 20 to $2000 \mathrm{ng} / \mathrm{mL}$ with the following regression equation: $y=0.6701 \times-0.0009(r=0.9998 ; n$ $=7$ ). The lower limit of quantification for voriconazole was $20 \mathrm{ng} / \mathrm{mL}$.

Moreover, approximate values for intra- and interday precision were $1.90 \%$ to $4.50 \%$ and $2.05 \%$ to $4.50 \%$, respectively. Accuracy expressed as bias ranged from- $8.74 \%$ to $-2.02 \%$ (Table 1 ).

\section{Pharmacokinetic properties}

The mean plasma concentration-time curves of two voriconazole formulations each administered a single $200 \mathrm{mg}$ oral dose (one $200 \mathrm{mg}$ tablet) to 19 healthy Chinese male volunteers are shown in the Figure 1. The primary PK parameters for both drugs are listed in Table 2.

The $90 \% \mathrm{Cls}$ of the ratios (test $v s$ reference) for the natural $\log (\ln )$ transformed $\mathrm{C}_{\max }, \mathrm{AUC}_{0-\mathrm{t}}$, and $\mathrm{AUC}_{0-\infty}$ are shown in Table 3. The $90 \%$ CIs for the ratios of $\mathrm{C}_{\max }, \mathrm{AUC}_{0-\mathrm{t}}$, and $\mathrm{AUC}_{0-\infty}$ were $77.3 \%$ to $122.7 \%$, $85.7 \%$ to $114.3 \%$, and $83.6 \%$ to $116.4 \%$, respectively, meeting the predetermined criteria for bioequivalence. The relative bioavailability 
Citation: Qiu X, Xiang R, Dai Q, Yang B, Xiong L, et al. (2012) Pharmacokinetics and Bioequivalence Evaluation of Two Voriconazole tablets: an Open-Label, Single-Dose, Randomized, Two-Way Crossover Study in Healthy Chinese Male Volunteers. J Bioequiv Availab 4: $044-047$. doi:10.4172/jbb.1000110

\begin{tabular}{|c|c|c|c|c|c|c|}
\hline \multirow[b]{2}{*}{$\begin{array}{c}\text { Theoretical } \\
\text { Concentration, } \\
\text { ng/mL }\end{array}$} & \multicolumn{3}{|c|}{ Intraday $(n=6)$} & \multicolumn{3}{|c|}{ Interday $(n=6)$} \\
\hline & $\begin{array}{c}\text { Actual } \\
\text { Concentration, } \\
\text { ng/mL }\end{array}$ & $\begin{array}{c}\text { RSD, } \\
\%\end{array}$ & $\begin{array}{c}\text { Bias, } \\
\%\end{array}$ & $\begin{array}{c}\text { Actual } \\
\text { Concentration, } \\
\text { ng/mL }\end{array}$ & $\begin{array}{c}\text { RSD, } \\
\%\end{array}$ & $\begin{array}{c}\text { Bias, } \\
\%\end{array}$ \\
\hline 50 & 48.99 & $2.85 \%$ & $-2.02 \%$ & 48.51 & $4.50 \%$ & $-2.98 \%$ \\
\hline 200 & 182.51 & $4.50 \%$ & $-8.74 \%$ & 185.89 & $4.01 \%$ & $-7.05 \%$ \\
\hline 1600 & 1518.71 & $1.90 \%$ & $-5.08 \%$ & 1525.90 & $2.05 \%$ & $-4.63 \%$ \\
\hline
\end{tabular}

$\mathrm{RSD}=$ relative standard deviation.

Table 1: Intra- and interday precision and bias of voriconazole in human plasma quality control samples prepared from healthy chinese male volunteers

of the test formulation was103.2\% (mean $\mathrm{AUC}_{0-t}$ ) and $104.8 \%$ (mean $\left.\mathrm{AUC}_{0-\infty}\right)$.

$\mathrm{C}_{\max }$, maximum plasma concentration; $\mathrm{T}_{\max }$, time to peak concentration; $\mathrm{AUC}_{0-\mathrm{t}}$, area under plasma concentration-time curve from zero to the last measurable concentration; $\mathrm{AUC}_{0-\infty}$, area under plasma concentration time curve from zero to infinity; $\mathrm{T}_{1 / 2}$, elimination half-life.

\section{Discussion}

In vitro, voriconazole has good antifungal activity against filamentous fungi. Animal experiments showed that voriconazole test preparation had equivalent antibacterial activity to the original voriconazole product. This study examined the PK properties and bioequivalence of two formulations of voriconazole in healthy Chinese adult male volunteers. The $90 \%$ CIs were completely contained within

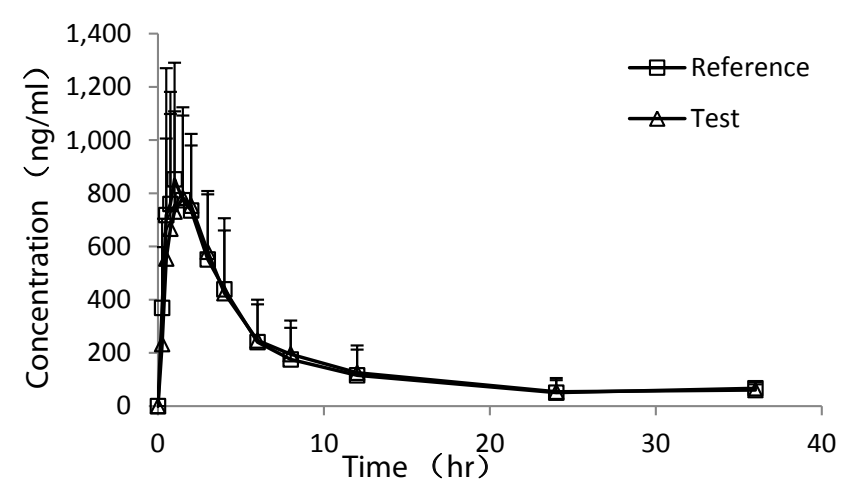

Figure 1: Mean plasma concentration-time profiles after administration of a single 200-mg dose of two voriconazole formulations in 19 healthy Chinese male volunteers.

\begin{tabular}{|l|c|c|}
\hline Parameter & Test & Vfend $^{\mathrm{TM}}$ (reference) \\
\hline $\mathrm{C}_{\max }(\mathrm{ng} / \mathrm{mL})$ & $925.73 \pm 356.11$ & $1040.25 \pm 448.93$ \\
\hline $\mathrm{T}_{\max }(\mathrm{h})$ & $1.57 \pm 0.98$ & $1.38 \pm 0.96$ \\
\hline $\mathrm{AUC}_{0-\mathrm{t}}\left(\mathrm{ng} / \mathrm{mL}^{*} \mathrm{~h}\right)$ & $5304.97 \pm 3072.25$ & $5141.63 \pm 2976.92$ \\
\hline $\mathrm{AUC}_{0-\infty}\left(\mathrm{ng} / \mathrm{mL}^{*} \mathrm{~h}\right)$ & $5783.21 \pm 3266.86$ & $5520.69 \pm 3148.42$ \\
\hline $\mathrm{T}_{1 / 2}(\mathrm{~h})$ & $7.80 \pm 2.16$ & $6.77 \pm 2.05$ \\
\hline
\end{tabular}

$\mathrm{C}_{\max }$, maximum plasma concentration; $\mathrm{T}_{\max }$, time to peak concentration; $A \cup \mathrm{C}_{0-\mathrm{t}}$, area under plasma concentration-time curve from zero to the last measurable concentration; $\mathrm{AUC}_{0-\infty}$, area under plasma concentration time curve from zero to infinity; $T_{1 / 2}$, elimination half-life

Table 2: Pharmacokinetic parameters of voriconazole after a single 200-mg oral dose in healthy chinese male volunteers $(N=19)$. Data are mean $\pm S D$.

\begin{tabular}{|c|c|c|}
\hline Parameter & Test/Reference Ratio & $90 \% \mathrm{Cl}$ \\
\hline $\ln \mathrm{C}_{\max }$ & 106.6 & $77.3-122.7$ \\
\hline InAUCO-t & 90.4 & $85.7-114.3$ \\
\hline 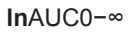 & 103.4 & 83.6-116.4 \\
\hline
\end{tabular}

Table3: Comparison of $90 \%$ Cls of natural $\log (\ln )$-transformed parameters for test/reference of voriconazole formulation in healthy chinese male volunteers.

the predefined bioequivalence criteria of $80 \%$ to $125 \%$ for AUC0-t, AUC0 $-\infty$ and $70 \%$ to $143 \%$ for $\mathrm{C}_{\max }$

AUC is accepted as an indicator of the extent of absorption, whereas $\mathrm{C}_{\max }$ and $\mathrm{T}_{\max }$ are considered estimates of the absorption rate. The study results revealed that the two formulations of voriconazole tablets had similar PK parameters and plasma concentration-time profiles among these healthy Chinese male volunteers. The PK parameters were not the same comparable to those published in previous study [5,8]. Gao Hongzhi et al. [5] reported that in a randomized-sequence, open-label, 2-period crossover study, voriconazole $200 \mathrm{mg}$ administrated to 20 healthy, fasting, Chinese volunteers produced a geometric mean $\mathrm{C}_{\max }$ of $1772.08 \mathrm{ng} / \mathrm{mL}, A U C 0-\infty$ of $9393.36 \mathrm{ng} / \mathrm{mL}^{\star}$ h. The parameters $\mathrm{C}_{\max }$ and $\mathrm{AUC} 0-\infty$ of the reference of voriconazole tablets in the present study were 1.7 times lower than that. Adolescents with low body weight may need to receive higher doses to match the adult exposures [9]. Gene polymorphism can affect the pharmacokinetic parameters of voriconazole [8-11]. As the age in the present study is lower than the population of the previous study, [5] the subjects may have a fast metabolism, which causes $\mathrm{C}_{\max }$ and $\mathrm{AUC} 0-\infty$ small; The volunteers were recruited in the southwest district of China, which should also be the reason of small $\mathrm{C}_{\max }$ and $\mathrm{AUC} 0-\infty$ in our study because of the possible gene polymorphism of the regional differences.

The mean $\mathrm{T}_{1 / 2}$ obtained in this study was 7.80 hours for the test product, which was comparable to that of the reference product at 6.77 hours. The individual half-lives ranged between 3.5 and 12.0 hours, so the 1-week washout period was sufficient because it was longer than 7 half lives for all volunteers. These results are consistent with PK characteristics previously reported. [5]

Our study was limited by using a single-dose design, including only healthy men, and studying participants only in the fasted state. Because healthy volunteers are not representative of a patient population, these results remain to be tested in patients.

\section{Conclusions}

In this small study in healthy Chinese adult male volunteers, a single $200 \mathrm{mg}$ dose of the tablet (test) of voriconazole met the regulatory 
Citation: Qiu X, Xiang R, Dai Q, Yang B, Xiong L, et al. (2012) Pharmacokinetics and Bioequivalence Evaluation of Two Voriconazole tablets: an Open-Label, Single-Dose, Randomized, Two-Way Crossover Study in Healthy Chinese Male Volunteers. J Bioequiv Availab 4: 044-047. doi:10.4172/jbb.1000110

criteria for bioequivalence to a single $200 \mathrm{mg}$ dose of the established tablet product (reference) based on the rate and extent of absorption. Both products were well tolerated.

\section{Acknowledgments}

The data analysis using Winnolin Version 5.2.1 was supported by Laboratory of Anesthesia and Critical Care Medicine West China Hospital, Sichuan University, China.

\section{References}

1. Espinel-Ingroff, A (1998) In vitro activity of the new triazole voriconazole (UK109,496) against opportunistic filamentous and dimorphic fungi and common and emerging yeast pathogens. J Clin Microbiol 36: 198-202.

2. Perfect JR, Marr KA, Walsh TJ, Greenberg RN, DuPont B, et al. (2003) Voriconazole treatment for less-common, emerging, or refractory fungal infections. Clin Infect Dis 36: 1122-1131.

3. Smith J, Andes D (2008) Therapeutic drug monitoring of antifungals: pharmacokinetic and pharmacodynamic considerations. Ther Drug Monit 30: 167-172.

4. Michael C, Bierbach U, Frenzel K, Lange T, Basara N, et al. (2010)Voriconazole pharmacokinetics and safety in immunocompromised children compared to adult patients. Antimicrob Agents Chemother 54: 3225-3232.

5. Gao Hong-zhi, Liang Yu-guang, Liu Zeyuan, Hu Jin-chao, Liu Ze-yuan (2007)
Bioequivalence of two voriconazole tablets in healthy volunteers. Chinese $\mathrm{J}$ New Drugs 16: 1133-1136.

6. State Food and Drug Administration of China [2003]. Guideline for Good Clinical Principles.

7. World Medical Association Declaration of Helsinki: Ethical Principles for Medical Research Involving Human Subjects, Adopted by the 18th WMA General Assembly, Helsinki, Finland, June 1964, and amended by the $52^{\text {nd }}$ WMA General Assembly, Edinburgh, Scotland, October7, 2000.

8. Huang Fei, Xia Chun-hua, Xiong Yu-qing (2011) Effect of cytochrome P450(CYP)3A5 CYP2C19 gene polymorphism on pharmacokinetic characteristics of voriconazole in Chinese healthy volunteers. Chin J Clin Pharmacol 27: 762-766.

9. Driscoll TA, Frangoul H, Nemecek ER, Murphey DK, Yu LC, et al. (2011) Comparison of pharmacokinetics and safety of voriconazole intravenous-tooral switch in immunocompromised children and healthy adults. Antimicrob Agents Chemother. 55: 5780-5789

10. Leveque D, Nivoix Y, Jehl F, Herbrecht R (2006) Clinical pharmacokinetics of voriconazole. Int J Antimicrobial Agents 27: 274-284.

11. Jue WL, Huilin T, Jingli D (2011) Systematic review of influence on pharmacokinetics of voriconazole on CYP2C19 genetic polymorphisms. Chin J Clin Pharmacol 27: 607-611. 